\title{
SCHÉMAS PRÉCOCES INADAPTÉS, TEMPÉRAMENT ET CARACTĖRE CHEZ DES PATIENTS PRÉSENTANT UNE DÉPENDANCE AUX SUBSTANCES PSYCHOACTIVES
}

Joëlle Berrewaerts, Romain Pallincourt, Géraldine Wildemeersch, Martin Desseilles et Philippe Kempeneers

Groupe d'études de psychologie | « Bulletin de psychologie »

2019/4 Numéro 562 | pages 243 à 257

ISSN 0007-4403

Article disponible en ligne à l'adresse :

https://www.cairn.info/revue-bulletin-de-psychologie-2019-4-page-243.htm

Distribution électronique Cairn.info pour Groupe d'études de psychologie.

(c) Groupe d'études de psychologie. Tous droits réservés pour tous pays.

La reproduction ou représentation de cet article, notamment par photocopie, n'est autorisée que dans les limites des conditions générales d'utilisation du site ou, le cas échéant, des conditions générales de la licence souscrite par votre établissement. Toute autre reproduction ou représentation, en tout ou partie, sous quelque forme et de quelque manière que ce soit, est interdite sauf accord préalable et écrit de l'éditeur, en dehors des cas prévus par la législation en vigueur en France. Il est précisé que son stockage dans une base de données est également interdit. 


\title{
Schémas précoces inadaptés, tempérament et caractère chez des patients présentant une dépendance aux substances psychoactives
}

\author{
BERREWAERTS Joëlle a \\ PALLINCOURT Romain ${ }^{\mathrm{b}}$ \\ WILDEMEERSCH Géraldine ${ }^{b}$ \\ DesseILles Martin a,b,* \\ KEMPENEERS Philippe ${ }^{b, c, *}$
}

\begin{abstract}
${ }^{a}$ Université de Namur, Département de psychologie, Faculté de médecine, 61 rue de Bruxelles, 5000 Namur, Belgique.

${ }^{\mathrm{b}}$ Clinique psychiatrique des Frères Alexiens, 68 rue du château de Ruyff, 4841 Henri-Chapelle, Belgique.

${ }^{\mathrm{c}}$ Université de Liège, Unité de recherche en psychologie et neurosciences cognitives, B33 boulevard du Rectorat, 4000 Liège, Belgique.

* Ces deux auteurs partagent la qualité d'auteur. Correspondance : Joëlle Berrewaerts, Université de Namur, Département de psychologie, 61 rue de Bruxelles, 5000 Namur, Belgique.

Courriel : joelle.berrewaerts@unamur.be
\end{abstract}

Texte reçu le 19 juillet 2016 et accepté le 14 janvier 2019

http://www.bulletindepsychologie.net
Résumé : Le questionnaire des schémas de Young (YSQ-S3) et celui des tempéraments et des caractères de Cloninger (TCI-R) ont été remplis par 49 femmes et 56 hommes présentant un trouble lié à l'utilisation d'une substance, tel que défini par le DSM-IV-TR. Pour $84,8 \%$ des personnes, le principal produit incriminé était l'alcool, soit à titre unique $(73,3 \%)$, soit en combinaison avec d'autres produits. Leur âge moyen est de 45,5 ans et s'étend de 21 à 64 ans. Pour le YSQ-S3, on relève une sur-activation de cinq schémas précoces inadaptés (SPI) dans l'échantillon : manque affectif, abandon/instabilité, abnégation, négativité/pessimisme et idéaux exigeants. Pour le TCI-R, on observe une surpondération des notes d'évitement du danger et une sous-pondération des notes d'autodétermination. D'un autre côté, des régressions linéaires montrent qu'une faible autodétermination, telle que mesurée par le TCI-R, permet de prédire une large palette de SPI. À un degré moindre, l'évitement du danger apparaît, également, comme un prédicteur significatif des SPI. Par ailleurs, la particularité d'un profil de réponse au TCI-R ne paraît guère à même de prédire un profil de réponse particulier au YSQ-S3.

Early maladaptive schemas, temperament, and character traits in individuals with substance use disorders

Abstract: The Young Schema Questionnaire (YSQ-S3) and Cloninger Temperament and Character Inventory (TCI-R) were completed by 49 women and 56 men with a substance use disorder as defined by DSM-IV-TR. For $84.8 \%$ of subjects, the main substance was alcohol, either alone $(73.3 \%)$ or in combination with other products. Their average age was 45.5 years, ranging from 21 to 64 years. For the YSQ-S3, there was an over-activation of five Early Maladaptive Schemas (EMS) in the sample: Emotional Deprivation, Abandonment/Instability, Self-sacrifice, Negativity/Pessimism, and Unrelenting Standards. For the TCI-R, an overweighting in the Harm Avoidance ratings and an underweighting in the Self-Directedness ratings were observed. On the other hand, linear regressions show that low Self-Directedness as measured by the TCI-R occurs in the explanation of a wide range of EMS. To a lesser degree, Harm Avoidance is also a significant explanator of EMS. Moreover, the TCI-R response profile seems unable to predict a particular response profile for the YSQ-S3. 


\section{INTRODUCTION}

\section{Personnalité et psychopathologie}

Un trait de personnalité désigne une caractéristique psychologique stable, disposant une personne à se comporter, à penser et à ressentir les choses d'une façon qui lui est particulière à travers des situations variées (Ashton, 2013 ; Hansenne, 2003). De semblables dispositions peuvent notamment avoir rapport à des conduites, des cognitions et des ressentis réputés pathologiques, si bien que de nombreux travaux en psychologie de la personnalité portent précisément sur la prédiction des psychopathologies. Certaines théories ou méthodes d'évaluation de la personnalité émanent, d'ailleurs, directement, de préoccupations cliniques. C'est le cas, par exemple, de l'Inventaire multiphasique de la personnalité du Minnesota (Hathaway, McKinley, 1943 ; Tellegen et coll., 2003) et, d'une certaine manière, du questionnaire des schémas de Young (1990), objet de cet article.

Certaines dispositions personnelles - des éléments de personnalité - peuvent ainsi être considérées comme des facteurs de vulnérabilité à la psychopathologie. Il existe, cependant, diverses manières d'identifier les dispositions - ou traits pertinents ; les théories de la personnalité sont, en effet, nombreuses.

Selon une perspective cognitive, les schémas liés à la conception de soi, c'est-à-dire les autoschémas, sont considérés comme des structures cognitives sous-jacentes stables, qui constituent un aspect important de la personnalité et contribuent à un comportement persistant et cohérent (Cervone, Pervin, 2008). Les théories socio-cognitives mettent surtout l'accent sur le rôle de l'environnement social dans l'acquisition des schémas (Halvorsen et coll., 2009). Dans la lignée de ces théories, Young (1990) défend l'idée que la non-satisfaction, au cours de l'enfance, de besoins affectifs fondamentaux donnera lieu au développement de schémas précoces inadaptés (SPI). Ces derniers sont des croyances inconditionnelles sur le monde, sur soi-même et sa relation aux autres.

Le modèle psychobiologique de Cloninger (1986), second objet de cet article, propose une perspective différente. Selon ce modèle, la personnalité est décrite comme le résultat de l'influence conjointe du tempérament, principalement déterminé par des facteurs génétiques et constitutionnels et du caractère, principalement déterminé par l'apprentissage et l'environnement psychosocial. Certaines combi- naisons de ces traits de tempérament et de caractère chez un même individu peuvent engendrer les catégories de personnalité pathologique décrites dans les classifications classiques.

Il est à noter que le modèle cognitiviste de Young a été essentiellement élaboré dans une heuristique clinique, en marge des courants de recherche dominants en psychologie de la personnalité qui, comme l'approche lexicale (Ashton et coll., 2004 ; Costa, McCrae, 1988 ; 1992 ; McCrae, Allik, 2002) ou la perspective psychobiologique (Cloninger, Svarkic, Przybeck, 1993), cherchent à élucider l'organisation de l'ensemble des dispositions des personnes. L'approche de Young porte spécifiquement sur des structures de pensée, tandis que, dans les autres démarches et notamment celle de Cloninger, la visée consiste davantage à rendre compte de la structuration générale de la personnalité humaine. En ce sens, les schémas cognitifs mis en avant par Young pourraient être considérés comme des épiphénomènes si l'on adopte le point de vue plus fondamentalement structurel de la démarche de Cloninger. De ce point de vue encore, même s'il s'agit d'entités psychologiques stables, les schémas ne pourraient pas, à proprement parler, être qualifiés de « traits de personnalité ». Quoi qu'il en soit, tant les SPI de Young que les traits et facteurs de personnalité identifiés dans le modèle de Cloninger, ont la prétention de prédire certains comportements, notamment des comportements pathologiques. Dans le modèle de Young, c'est, d'ailleurs, très explicitement, la détection de propensions psychopathologiques qui est l'objectif visé. De fait, chaque courant de recherche a, de son côté, établi des corrélations entre certains tableaux psychopathologiques, d'une part, et des configurations psychologiques stables, d'autre part, des configurations dispositionnelles conçues, tantôt comme des traits de personnalité (Cloninger, Svrakic, Przybeck, 2006 ; Loftus, Garno, Jaeger, Malhotra, 2008), tantôt comme des schémas cognitifs (Lee, Taylor, Dunn, 1999 ; Schmidt, Joiner, Young, Telch, 1995).

Indépendamment de la manière dont Young a théorisé l'épigenèse des schémas cognitifs inadaptés, il semble cohérent de soutenir que des traits de personnalité, tels que conçus par Cloninger, peuvent participer à leur développement.

\section{Personnalité et troubles liés aux substances psychoactives}

La personnalité est un facteur susceptible de jouer un rôle dans la prédisposition, la précipitation ou la perpétuation des comportements d'abus ou de 
dépendance (Elkins, King, McGue, Iacono, 2006 ; Le Bon et coll., 2004). De nombreuses recherches ont mis en évidence un taux élevé de troubles de la personnalité parmi les patients dépendants à l'alcool ou à d'autres substances psychotropes (BowdenJones et coll., 2004 ; Compton, Conway, Stinson, Colliver, Grant, 2005 ; Craig, 2000 ; Grant et coll., 2004 ; Preuss et coll. 2009 ; Verheul, 2001).

L'approche de Cloninger et celle de Young peuvent, toutes deux, contribuer à une meilleure compréhension des antécédents développementaux pertinents dans la dépendance aux substances. Tant Young, Klosko, Weishaar, (2003) que Cloninger, Svrakic et Svrakic (1997) ont proposé des marqueurs de vulnérabilité pour la dépendance aux substances dans leurs modèles respectifs.

En 1998, Ball et Young ont émis 1'hypothèse que les individus présentant des critères d'abus de substance manifestaient une sur-activation des SPI de manque de contrôle de soi ou de droits personnels/dominance. Ils ont également suggéré que les facteurs déclenchant l'abus et l'utilisation de substance constituaient des styles d'adaptation dysfonctionnels, utilisés pour éviter ou compenser l'activation de SPI d'abandon/instabilité, de méfiance/abus, de manque affectif, d'imperfection/honte, d'isolement/aliénation, d'échec, de surprotection/personnalité atrophiée, de dépendance/incompétence, de surcontrôle, d'idéaux exigeants et de punition.

Plusieurs recherches ont examiné la suractivation effective des SPI parmi des personnes présentant des critères d'abus ou de dépendance aux substances. Shorey, Stuart et Anderson (2013, 2014) ont observé des scores relativement élevés à de nombreux SPI parmi des jeunes hommes et femmes en recherche d'un traitement pour abus de substances, en comparaison de ce qui était observé chez des sujets contrôles. De même, Brotchie, Meyer, Copello, Kidney, Waller (2004) ont observé que des patients abuseurs de substances (alcool et opiacés) rapportaient moins de schémas sains que des sujets contrôles. Dans une étude de Roper, Dickson, Tinwell, Booth, McGuire (2010), des personnes dépendantes à l'alcool présentaient des scores plus élevés que des sujets contrôles à 12 SPI sur 15. Dans une étude de Decouvelaere, Graziani, Gackiere-Eraldi, Rusinek, Hautekèete (2002), il est apparu qu'à l'âge adulte, les sujets alcoolo-dépendants présentaient des schémas précoces mal adaptés plus actifs que des sujets contrôles, pour 12 des 13 schémas étudiés. Dans une étude de cas cliniques, Grebot et Marchand (2008) ont observé une utilisation importante du schéma cognitif « manque affectif » chez trois étudiants grands consommateurs de cannabis. Dans le cadre du jeu pathologique, une pathologie également considérée comme un problème de dépendance, Katona et Körmendi (2012) ont observé plusieurs SPI actifs parmi 23 joueurs membres d'un groupe d'auto-assistance, en comparaison de sujets contrôles. Ces études indiquent que les schémas de Young peuvent refléter une vulnérabilité cognitive chez les sujets dépendants.

Cloninger, Svrakic et Svrakic (1997) ont également mis en évidence sur la base d'analyses préliminaires d'un échantillon de la population que les différences individuelles dans la structure et le développement de la personnalité avaient une forte influence sur le risque de toutes les formes de psychopathologie, y compris l'abus de substances. Selon Cloninger, les traits de tempérament jouent un rôle central dans les addictions. Pour ce qui est de l'alcoolisme, Cloninger (1987a) émet l'hypothèse, dès 1987, que celui-ci procéderait d'au moins deux grandes tendances : à côté d'un profil dit «alcoolisme de type II », caractérisé par un niveau haut de recherche de nouveauté et des niveaux bas d'évitement du danger et de dépendance à la récompense, existerait un profil dit " de type I », caractérisé par de hauts niveaux d'évitement du danger. L'un serait de début précoce, essentiellement masculin et souvent accompagné de multiples comportements impulsifs, l'autre serait de début tardif, aussi bien masculin que féminin, et s'accompagnerait de problèmes anxieux (Cloninger, 1987a ; Cloninger et coll., 1988).

De manière générale, il semble que la recherche de nouveauté soit la principale dimension de tempérament à être liée à la dépendance et à l'abus de substances. En effet, de nombreuses études montrent que ce trait est plus prononcé chez les personnes dépendantes aux substances que chez des sujets témoins, dans plusieurs types de dépendance : dépendance à l'alcool (Basiaux et coll., 2001 ; Howard, Kivlahan, Walker, 1997 ; Le Bon, et coll., 2004a ; Lukasiewicz et coll., 2008; Masse, Tremblay, 1997 ; Ravaja, Keltikangas-Jarvinen, 2001), dépendance au cannabis (Lukasiewicz et coll. 2008 ; van Ammers, Sellman, Mulder, 1997), aux opiacés (Abbate-Daga, Amianto, Rogna, Fassino, 2007 ; Cohen et coll., 2005, Fassino, Daga, Delsedime, Rogna, Boggio, 2004 ; Le Bon et coll., 2004 ; Vukov, Baba-Milkic, Lecic, Mijalkovic, Marinkovic, 1995), à la cocaïne (Herrero et coll., 
2008 ; Prisciandaro, Korte, McRae-Clark, Brady, 2012) et à la métamphétamine (Hosak, Preiss, Halir, Cermakova, Csemy, 2004).

La littérature présente parfois aussi la recherche de nouveauté comme un prédicteur de la consommation de substances psychoactives au sein de populations non cliniques d'adolescents et d'étudiants universitaires (Chakroun, Doron, Swendsen, 2004 ; George, Connor, Gullo, Young, 2010 ; Masse, Tremblay, 1997 ; Wills, Vaccaro, McNamara, 1994).

Pour le trait de tempérament d'évitement du danger, les données semblent moins univoques. Certaines études montrent une relation entre l'utilisation de substances psychoactives et des scores plus faibles en évitement du danger (Wills et coll., 1994 ; Skeel, Pilarshi, Pytlak, Neudecker, 2008) ; d'autres, en revanche, montrent que les personnes dépendantes obtiennent plutôt des scores élevés (Fassino et coll., 2004 ; Hosak, Preiss, Halir, Cermakova, Csemy, 2004 ; Abbate-Daga et coll., 2007).

Les personnes dépendantes aux substances semblent, également, avoir des traits de caractère différents des personnes non dépendantes : elles obtiennent généralement des scores moins élevés en autodétermination (Abbate-Daga et coll., 2007 ; Basiaux et coll., 2001 ; Cohen et coll., 2005 ; Fassino et coll., 2004 ; Herrero et coll., 2008 ; Hosak, Preiss, Halir, Cermakova, Csemy, 2004 ; Le Bon et coll., 2004) et en coopération (Abbate-Daga et coll., 2007; Herrero et coll., 2008) et des scores plus élevés en transcendance (Abbate-Daga et coll., 2007 ; Fassino et coll., 2004 ; Herrero et coll., 2008 ; Hosak et coll., 2004 ; Le Bon et coll., 2004).

\section{Objectif de l'étude}

Le but de cette étude est de rechercher les facteurs de vulnérabilité pour la dépendance aux substances, à partir de deux lignes distinctes de tradition de recherche clinique, soit, d'une part, les schémas précoces inadaptés (SPI) tels qu'évalués par le Questionnaire des schémas de Young, $3^{\text {e }}$ version, forme abrégée (YSQ-S3, Young et coll., 2003) et, d'autre part, le tempérament et le caractère, tels qu'évalués par l'Inventaire révisé des tempéraments et des caractères (TCI-R ; Cloninger, 1999).

En fonction des résultats des recherches précédentes, nous faisons l'hypothèse que :

- les patients dépendants aux substances présenteront plusieurs schémas précoces inadaptés, et spécifiquement de type abandon/instabilité, méfiance/abus, manque affectif, imperfection/ honte, isolement/aliénation, échec, surprotection/ personnalité atrophiée, dépendance/incompétence, surcontrôle, idéaux exigeants et punition ;

- les patients dépendants aux substances présenteront des scores supérieurs aux normes pour les dimensions de recherche de nouveauté et de transcendance, et inférieurs aux normes pour les dimensions d'autodétermination et de coopération ;

- la connaissance des résultats au TCI-R permettra de prédire, au moins en partie, les résultats au YSQ-S3.

\section{MÉTHODE}

\section{Participants et procédure}

L'analyse a été faite sur un échantillon de 105 patients recrutés au sein d'un hôpital de jour psychiatrique belge francophone.

De 2009 à 2012, des examens psychométriques ont été réalisés en routine auprès de tout patient accueilli au centre de jour et présentant une problématique connue liée à l'utilisation de psychotropes. Les questionnaires de Cloninger (TCI-R) et de Young (YSQ-S3) ont été remplis par 49 femmes et 56 hommes, soit 105 personnes répondant aux critères diagnostiques de dépendance à une substance $(\mathrm{N}=98)$ ou d'abus d'une substance $(\mathrm{N}=7)$, tels que définis par le DSM-IV-TR. Cet échantillon de 105 sujets représentait $26,85 \%$ de la population totale des personnes admises à l'hôpital de jour, toutes pathologies confondues, de début 2009 à fin 2012. Cinq patients $(4,54 \%)$, diagnostiqués dépendants, n'ont pas été inclus dans l'échantillon en raison de leur départ précoce, d'un problème de langue ou de problèmes cognitifs ou psychoaffectifs hypothéquant la bonne compréhension des questionnaires proposés.

L'âge moyen des sujets évalués s'établissait à 45,5 ans (46 ans pour les femmes, 45 ans pour les hommes), avec des extrêmes de 21 et 64 ans. Pour 89 sujets $(84,76 \%)$, le principal produit incriminé était l'alcool, soit à titre unique ( $\mathrm{N}=77 ; 73,33 \%)$, soit en combinaison avec d'autres produits : benzodiazépines $(\mathrm{N}=7)$, cannabis $(\mathrm{N}=3)$, opiacés $(\mathrm{N}=$ 1) ou cocaïne $(\mathrm{N}=1)$. Cinq personnes $(4,76 \%)$ présentaient une dépendance aux seules benzodiazépines, trois $(2,86 \%)$ aux opiacés, deux $(1,90 \%)$ au cannabis et une $(0,95 \%)$ à la cocaïne. Pour cinq autres personnes $(4,76 \%)$, admises pour un problème de dépendance aux substances, le type de produit problématique n'a pu être déterminé avec précision en raison de leur abandon précoce de l'hospitalisation de jour. 
L'évaluation diagnostique des patients a été fondée sur les informations transmises par les structures psychiatriques externes, sises en amont du centre de jour, complétées par des investigations conduites par une équipe interne de psychologues et psychiatres. Les diagnostics ont été libellés dans les termes du DSM-IV-TR. Il s'agit d'estimations diagnostiques minimales, dans la mesure où, seules, les problématiques explicitement rapportées par les patients ou leur entourage ont été prises en compte. Soixantequatre patients sur $105(60,95 \%)$ présentaient clairement une ou plusieurs autres psychopathologies associées au problème d'utilisation de substances. Par ordre décroissant d'importance, on recense $32(30,48 \%)$ troubles dépressifs, dont 5 de nature bipolaire ; $24(22,86 \%)$ troubles de la personnalité du groupe B, essentiellement de type personnalité limite ; $16(15,24 \%)$ troubles anxieux, dont 2 troubles obsessionnels-compulsifs ; 6 (5,71\%) troubles de l'adaptation avec symptômes anxiodépressifs/ou perturbations des conduites ; $5(4,76 \%)$ troubles de la personnalité du groupe C ; $2(1,90 \%)$, troubles du contrôle des impulsions ; un $(0,95 \%)$ trouble de la personnalité schizoïde, un trouble de la personnalité non-spécifié et un déficit de l'attention avec hyperactivité. Le nombre de difficultés ainsi recensées excède le nombre des 64 patients, car plusieurs d'entre eux présentaient plus de deux diagnostics.

\section{Mesures}

\section{$Y S Q-S 3$}

Le YSQ-S3 (Young Schema Questionnaire Short form Third edition) (Young et coll., 2003) est un inventaire auto-administré, comportant 90 items et ayant pour but d'évaluer l'allégeance relative des sujets à 18 schémas précoces inadaptés. Les 90 items sont regroupés en 18 sous-échelles correspondant aux 18 SPI. Ces 18 schémas, eux-mêmes regroupés en 5 domaines, sont répertoriés dans la colonne de gauche du tableau 1.

La version française du YSQ-S3 a été traduite par Pascal et Cousineau (Young, Pascal, Cousineau, 2005). Le niveau d'allégeance d'un sujet à chacun des 18 schémas dysfonctionnels du modèle y est évalué à l'aide de 5 affirmations, assorties d'une échelle de réponse graduée de 1 (« cela est complètement faux pour moi ») à 6 (« cela me décrit parfaitement »). Conformément aux recommandations de Young, Klosko et Weishaar (2005), l'allégeance à un schéma dysfonctionnel a été mesurée par la proportion de réponses 5 (« le plus souvent vrai pour moi ») ou 6 (« cela me décrit parfaitement ») aux cinq propositions qui dénotent la présence du schéma en question. Cette mesure est exprimée en pourcentage, la note attendue d'un sujet réputé sain étant zéro. Plus les scores sont élevés, plus ils attestent la présence du SPI chez le sujet qui répond au questionnaire.

\section{$T C I-R$}

\section{Le TCI-R (Temperament and Character Inventory-} Revised) (Cloninger, 1999) est un auto-questionnaire comportant 240 propositions, assorties d'échelles de réponse en 5 points, allant d' « absolument faux » à « absolument vrai ». Il a pour but d'évaluer les différences individuelles sur les dimensions de base du tempérament et du caractère. Il mesure quatre dimensions de tempérament (recherche de nouveauté, évitement du danger, dépendance à la récompense et persistance) et trois dimensions de caractère (autodétermination, coopération et transcendance). Chacune des dimensions de tempérament et de caractère est subdivisée en plusieurs sous-dimensions ou traits de personnalité, dont le détail figure dans la colonne gauche du tableau 2 .

La version française du TCI-R, utilisée dans cette étude, a été traduite par Pélissolo et Lépine (2000). Les normes publiées par Hansenne, Delhez, Cloninger (2005) ont servi de référence. Elles diffèrent quelque peu pour les hommes et pour les femmes. Les notes obtenues par les sujets ont été exprimées, d'une part, en notes brutes et, d'autre part, en notes standards de moyenne 50 et d'écart-type 10 , en référence aux normes d'Hansenne et coll. (2005).

\section{Analyses statistiques}

Le YSQ-S3 n'ayant fait l'objet d'aucune étude normative sur des populations de référence, l'activation relative de SPI au sein de notre échantillon a dû être appréciée sur la base de considérations cliniques. Si, d'un point de vue clinique, il est attendu que la note d'activation d'un SPI ne s'écarte guère de zéro chez un sujet réputé sain, il semble également cohérent de considérer cette situation comme idéale-typique, aussi avons-nous tenu compte de marges d'appréciation. Dans cette étude, les seuils de signification clinique ont ainsi été fixés à $20 \%$ et à $30 \%$, désignant deux niveaux de sévérité : les valeurs comprises entre $20 \%$ et $30 \%$ désignent un niveau « modéré » d'activation d'un SPI et les valeurs supérieures à $30 \%$ un niveau « marqué ».

Les écarts des sujets de notre échantillon aux normes du TCI-R, telles que publiées par Hansenne, Delhez, Cloninger (2005), ont été évalués à l'aide de tests $t$. 
La capacité des données issues du TCI-R à prédire les variations des SPI, tels que mesurés par le YSQ-S3, a été évaluée en deux phases : $1^{\circ}$ des corrélations (Spearman) ont d'abord été calculées entre chaque SPI (critères) et les notes du TCI-R (variables prédictives) ; $2^{\circ}$ les variables du TCI-R significativement $(\mathrm{p}>0,05)$ corrélées à un SPI donné, ont ensuite été introduites dans un modèle de régression de ce SPI sur ses corrélats. Des régressions pas-à-pas, par sélection incrémentielle ascendante (stepwise forward), ont ainsi été effectuées pour chaque critère, en prenant comme prédicteurs tantôt les dimensions (tempéraments et caractères) tantôt les sous-dimensions (traits) du TCI-R.

\section{RÉSULTATS}

\section{Structure des réponses au YSQ-S3}

Le tableau 1 rapporte les notes moyennes obtenues par les sujets aux 18 SPI, mesurés par le YSQ-S3. Les résultats sont exprimés en pourcentages de réponses 5 (« le plus souvent vrai pour moi ») ou 6 ( « cela me décrit parfaitement») aux cinq propositions, qui dénotent la présence du SPI considéré. Si l'on retient un seuil de signification clinique supérieur à $20 \%$, comme l'ont fait Pallincourt et coll. (2009), il apparaît que 14 SPI sur 18 peuvent être considérés comme activés, au moins modérément. Seuls, imperfection/honte, échec, dépendance/ incompétence, personnalité atrophiée et contrôle de soi insuffisant n'apparaissent pas significativement activés. Au seuil de signification clinique de $30 \%$, cinq SPI sont nettement suractivés : manque affectif, abandon/instabilité, abnégation, négativité/ pessimisme et idéaux exigeants.

Il convient, d'un autre côté, de relever l'importance des indices de dispersion (ET), lesquels témoignent d'une forte hétérogénéité au sein de l'échantillon.

\section{Structure des réponses au TCI-R}

Le tableau 2 présente les scores moyens obtenus par les sujets aux 7 dimensions du TCI-R, ainsi qu'aux différentes sous-dimensions.

\section{Séparations et rejets}

1. Manque affectif (emotional deprivation - ed)

$35,92(34,23)^{\star *}$

2. Abandon/instabilité (abandonment/instability - ab)

$33,59(34,32)^{\star \star}$

3. Méfiance/abus (mistrust/abuse - ma)

$24,27(30,38)^{\star}$

4. Isolement social (social isolation - is)

$25,63(29,83)^{\star}$

5. Imperfection/honte (defectiveness/shame)

$18,25(27,46)$

II. Manque d'autonomie et de performance

6. Echec (failure - fa)

7. Dépendance/incompétence (dependence/incompetence - di)

8. Peur du danger ou de la maladie (vulnerability to harm or illness - vh)

9. Fusionnement/personnalité atrophiée (enmeshment/underdeveloped self - em)

\section{Manque de limites}

10. Droits personnels exagérés/grandeur (entitlement/grandiosity - et)

11. Contrôle de soi/autodiscipline insuffisants (insufficient self-control - is)

\section{Dépendance aux autres}

12. Assujettissement (subjugation - sb)

13. Abnégation (self-sacrifice - ss)

14. Recherche d'approbation et de reconnaissance (approval seeking - as)

\section{Hypervigilance et inhibition}

15. Négativité/pessimisme (negativity/pessimism - np)

16. Surcontrôle émotionnel (emotional inhibition - ei)

17. Idéaux exigeants/critique excessive (unrelenting standards - us) 


\begin{tabular}{|c|c|c|c|c|}
\hline \multirow[b]{2}{*}{ TCI-R } & \multirow{2}{*}{$\begin{array}{c}\text { notes brutes } \\
\qquad M(E T)\end{array}$} & \multicolumn{2}{|c|}{$\begin{array}{l}\text { écarts à la norme } \\
\qquad(\mathrm{dl}=104)^{\star}{ }^{\star},{ }^{\star}\end{array}$} & \multirow{2}{*}{$\begin{array}{c}\text { notes } \mathbf{S}^{\star} \\
M\end{array}$} \\
\hline & & $t$ & $p$ & \\
\hline NS1 (excitabilité exploratoire) & $30,14(6,47)$ & & & 48,19 \\
\hline NS2 (impulsivité) & $25,26(5,59)$ & 2,3 & $<0,03$ & 52,41 \\
\hline NS3 (extravagance) & $29,31(7,68)$ & 4,69 & $<0,0001$ & 55,63 \\
\hline NS4 (manque d'ordre) & $18,31(4,4)$ & & & 47,61 \\
\hline Recherche de nouveauté - NS & $103,03(16,19)$ & & & 52,04 \\
\hline HA1 (inquiétude anticipatoire) & $35,98(8,8)$ & 6,27 & $<0,0001$ & 58,9 \\
\hline HA2 (peur de l'incertain) & $23,50(6,45)$ & & & 53,1 \\
\hline HA3 (timidité avec les inconnus) & $22,80(6,63)$ & 3,4 & $<0,001$ & 53,85 \\
\hline HA4 (fatigabilité) & $26,56(7,50)$ & 8,42 & $<0,0001$ & 61,5 \\
\hline Évitement du danger - HA & $109,06(24,14)$ & 6,39 & $<0,0001$ & 59,08 \\
\hline RD1 (sentimentalité) & $29,47(4,59)$ & & & 51,89 \\
\hline RD2 (chaleureux) & $32,68(7,45)$ & $-2,37$ & $<0,02$ & 47,62 \\
\hline RD3 (attachement) & $17,22(5,37)$ & $-4,93$ & $<0,0001$ & 45,05 \\
\hline RD4 (dépendance) & $19,20(3,83)$ & & & 51,68 \\
\hline Dépendance à la récompense - RD & $98,66(15,99)$ & & & 48,3 \\
\hline PS1 (courageux) & $29,03(6,83)$ & & & 47,98 \\
\hline PS2 (travailleur) & $25,82(6,37)$ & $-3,35$ & $<0,01$ & 45,64 \\
\hline PS3 (ambitieux) & $30,93(7,25)$ & $-2,79$ & $<0,01$ & 46,21 \\
\hline PS4 (perfectionnisme) & $25,06(6,26)$ & $-2,69$ & $<0,01$ & 46,72 \\
\hline Persistance - PS & $110,83(23,90)$ & $-2,99$ & $<0,01$ & 46,07 \\
\hline SD1 (responsabilité) & $24,67(6,94)$ & $-6,65$ & $<0,0001$ & 41,35 \\
\hline SD2 (buts dans la vie) & $18,00(5,49)$ & $-7,1$ & $<0,0001$ & 40,25 \\
\hline SD3 (ressources personnelles) & $15,57(4,26)$ & $-5,36$ & $<0,0001$ & 43,03 \\
\hline SD4 (acceptation de soi) & $33,92(8,03)$ & & & 51,13 \\
\hline SD5 (seconde nature éclairée) & $32,84(7,93)$ & $-6,8$ & $<0,0001$ & 40,7 \\
\hline Autodétermination - SD & $125,00(23,19)$ & $-6,67$ & $<0,0001$ & 41,32 \\
\hline C1 (tolérance sociale) & $29,42(5,02)$ & $-2,21$ & $<0,03$ & 48,83 \\
\hline C2 (empathie) & $17,60(3,64)$ & & & 48,47 \\
\hline C3 (solidarité) & $28,53(4,39)$ & $-5,52$ & $<0,0001$ & 44,51 \\
\hline C4 (indulgence) & $25,19(7,48)$ & & & 49,98 \\
\hline C5 (générosité désintéressée) & $28,55(4,72)$ & & & 48,63 \\
\hline Coopération - C & $129,12(18,27)$ & $-2,79$ & $<0,01$ & 47,36 \\
\hline ST1 (négligence de soi) & $31,24(6,60)$ & 2,08 & $<0,05$ & 52,01 \\
\hline ST2 (identification transpersonnelle) & $21,22(5,65)$ & & & 49,73 \\
\hline ST3 (acceptation spirituelle) & $19,70(6,64)$ & & & 50,32 \\
\hline Transcendance - ST & $72,15(15,25)$ & & & 50,82 \\
\hline
\end{tabular}

Tableau 2. Scores moyens aux dimensions et sous-dimensions du TCl-R ( $\mathrm{n}$ valide $=105$ ).

* références : Hansenne, Delhez et Cloninger (2005) ; ${ }^{\star *} t$ et $p$ rapportés lorsque $p<0,05$. 
Les scores apparaissent significativement supérieurs aux normes pour la dimension évitement du danger et inférieurs aux normes pour les dimensions persistance, autodétermination et coopération.

Pour les dimensions recherche de nouveauté, dépendance à la récompense et transcendance, les scores globaux ne diffèrent pas significativement des normes. Toutefois, l'analyse des résultats aux sous-dimensions permet d'observer que les sujets obtiennent, en moyenne, des scores signi- ficativement supérieurs aux normes à deux sousdimensions de la recherche de nouveauté : NS2 (impulsivité) et NS3 (extravagance). Ils obtiennent également des scores significativement inférieurs aux normes à deux sous-dimensions de la dépendance à la récompense : RD2 (ouvert à la communication chaleureuse) et RD3 (attachement). Pour la dimension transcendance, les patients obtiennent des scores significativement plus élevés à la sousdimension ST1 (négligence de soi).

\begin{tabular}{|c|c|c|c|c|c|}
\hline Schémas & $\begin{array}{l}\mathrm{R} 2 \\
\text { ajusté }\end{array}$ & dimensions retenues & $F$ & $p$ & $\beta$ \\
\hline Manque affectif (ed) & 0,09 & HA (Évitement du danger) & 11,59 & $<0,001$ & 0,32 \\
\hline Abandon/instabilité (ab) & 0,22 & SD (Auto-détermination) & 29,36 & $<0,0001$ & $-0,47$ \\
\hline Méfiance/abus (ma) & 0,16 & SD (Auto-détermination) & 19,96 & $<0,0001$ & $-0,41$ \\
\hline \multirow[t]{3}{*}{ Isolement social (si) } & 0,32 & SD (Auto-détermination) & 7,26 & $<0,01$ & $-0,3$ \\
\hline & & HA (Évitement du danger) & 5,38 & $<0,03$ & 0,26 \\
\hline & & $\begin{array}{l}\mathrm{RD} \text { (Dépendance à la } \\
\text { récompense) }\end{array}$ & 4,16 & $<0,05$ & $-0,17$ \\
\hline Imperfection/honte (ds) & 0,27 & SD (Auto-détermination) & 38,21 & $<0,0001$ & $-0,52$ \\
\hline \multirow[t]{2}{*}{ Échec (fa) } & 0,29 & HA (Évitement du danger) & 8,57 & $<0,005$ & 0,33 \\
\hline & & SD (Auto-détermination) & 5,38 & $<0,03$ & $-0,26$ \\
\hline \multirow[t]{2}{*}{$\begin{array}{l}\text { Dépendance/incompétence } \\
\text { (di) }\end{array}$} & 0,25 & SD (Auto-détermination) & 11,29 & $<0,005$ & $-0,33$ \\
\hline & & PS (Persistance) & 6,75 & $<0,02$ & $-0,26$ \\
\hline $\begin{array}{l}\text { Peur du danger ou de la } \\
\text { maladie (vh) }\end{array}$ & 0,21 & SD (Auto-détermination) & 28,29 & $<0,0001$ & $-0,47$ \\
\hline $\begin{array}{l}\text { Fusionnement/personnalité } \\
\text { atrophiée (em) }\end{array}$ & 0,13 & SD (Auto-détermination) & 16,19 & $<0,001$ & $-0,37$ \\
\hline $\begin{array}{l}\text { Droits personnels exagérés/ } \\
\text { grandeur (et) }\end{array}$ & 0,22 & SD (Auto-détermination) & 29,05 & $<0,0001$ & $-0,47$ \\
\hline \multirow[t]{2}{*}{$\begin{array}{l}\text { Contrôle de soi/autodiscipline } \\
\text { insuffisants (is) }\end{array}$} & 0,34 & SD (Auto-détermination) & 23,15 & $<0,0001$ & $-0,45$ \\
\hline & & PS (Persistance) & 5,23 & $<0,03$ & $-0,21$ \\
\hline Assujettissement (sb) & 0,28 & SD (Auto-détermination) & 40,35 & $<0,0001$ & $-0,53$ \\
\hline Abnégation (ss) & 0,1 & C (Coopération) & 12,51 & $<0,001$ & 0,33 \\
\hline $\begin{array}{l}\text { Recherche d'approbation et } \\
\text { de reconnaissance (as) }\end{array}$ & 0,11 & SD (Auto-détermination) & 14,24 & $<0,001$ & $-0,35$ \\
\hline Négativité/pessimisme (np) & 0,16 & HA (Évitement du danger) & 20,45 & $<0,0001$ & 0,41 \\
\hline \multirow[t]{2}{*}{ Sur-contrôle émotionnel (ei) } & 0,19 & $\begin{array}{l}\mathrm{RD} \text { (Dépendance à la } \\
\text { récompense) }\end{array}$ & 15,01 & $<0,001$ & $-0,36$ \\
\hline & & HA (Évitement du danger) & 4,87 & $<0,03$ & 0,2 \\
\hline $\begin{array}{l}\text { Idéaux exigeants/critique } \\
\text { excessive (us) }\end{array}$ & 0,1 & PS (Persistance) & 12,85 & $<0,001$ & 0,34 \\
\hline Punition (pu) & 0,06 & HA (Évitement du danger) & 7,79 & $<0,01$ & 0,27 \\
\hline
\end{tabular}

Tableau 3. Régression du YSQ-S3 sur les dimensions (tempéraments et caractères) du TCI-R. 


\begin{tabular}{|c|c|c|c|c|c|}
\hline Schémas & $\begin{array}{l}\mathrm{R} 2 \\
\text { ajusté }\end{array}$ & sous-dimensions retenues & $F$ & $p$ & $\beta$ \\
\hline Manque affectif (ed) & 0,1 & HA4 (Fatigabilité) & 12,6 & $<0,001$ & 0,33 \\
\hline \multirow[t]{2}{*}{ Abandon/instabilité (ab) } & \multirow[t]{2}{*}{0,23} & HA1 (Inquiétude anticipatoire) & 25,53 & $<0,0001$ & 0,44 \\
\hline & & ST1 (Négligence de soi) & 6,93 & $<0,01$ & 0,23 \\
\hline \multirow[t]{2}{*}{ Méfiance/abus (ma) } & \multirow[t]{2}{*}{0,21} & SD1 (Responsabilité) & 9,28 & $<0,005$ & $-0,3$ \\
\hline & & HA4 (Fatigabilité) & 5,93 & $<0,02$ & 0,24 \\
\hline \multirow[t]{3}{*}{ Isolement social (si) } & \multirow[t]{3}{*}{0,32} & RD2 (Chaleureux) & 7,29 & $<0,01$ & $-0,22$ \\
\hline & & HA4 (Fatigabilité) & 5,84 & $<0,02$ & 0,27 \\
\hline & & SD3 (Ressources personnelles) & 5,46 & $<0,03$ & $-0,25$ \\
\hline \multirow[t]{3}{*}{ Imperfection/honte (ds) } & \multirow[t]{3}{*}{0,29} & SD3 (Ressources personnelles) & 9,48 & $<0,005$ & $-0,33$ \\
\hline & & SD4 (Acceptation de soi) & 5,42 & $<0,03$ & $-0,2$ \\
\hline & & HA3 (Timidité avec les inconnus) & 4,5 & $<0,04$ & 0,23 \\
\hline \multirow[t]{3}{*}{ Échec (fa) } & \multirow[t]{3}{*}{0,3} & HA4 (Fatigabilité) & 11,95 & $<0,001$ & 0,33 \\
\hline & & SD5 (Seconde nature éclairée) & 9,04 & $<0,005$ & $-0,29$ \\
\hline & & RD1 (Sentimentalité) & 4,04 & $<0,05$ & 0,17 \\
\hline \multirow[t]{2}{*}{ Dépendance/incompétence (di) } & \multirow[t]{2}{*}{0,28} & PS2 (Travailleur) & 16,13 & $<0,001$ & $-0,36$ \\
\hline & & SD1 (Responsabilité) & 10,42 & $<0,005$ & $-0,29$ \\
\hline \multirow[t]{2}{*}{$\begin{array}{l}\text { Peur du danger } \\
\text { ou de la maladie (vh) }\end{array}$} & \multirow[t]{2}{*}{0,32} & HA1 (Inquiétude anticipatoire) & 27,9 & $<0,0001$ & 0,43 \\
\hline & & SD4 (Acceptation de soi) & 18,12 & $<0,0001$ & $-0,35$ \\
\hline \multirow{2}{*}{$\begin{array}{l}\text { Fusionnement/personnalité } \\
\text { atrophiée (em) }\end{array}$} & \multirow[t]{2}{*}{0,19} & SD1 (Responsabilité) & 15,25 & $<0,001$ & $-0,36$ \\
\hline & & ST1 (Négligence de soi) & 5,41 & $<0,03$ & 0,21 \\
\hline \multirow{2}{*}{$\begin{array}{l}\text { Droits personnels exagérés/ } \\
\text { grandeur (et) }\end{array}$} & \multirow[t]{2}{*}{0,29} & SD4 (Acceptation de soi) & 25,13 & $<0,0001$ & $-0,42$ \\
\hline & & PS1 (Courageux) & 20,85 & $<0,0001$ & $-0,38$ \\
\hline \multirow{4}{*}{$\begin{array}{l}\text { Contrôle de soi/autodiscipline } \\
\text { insuffisants (is) }\end{array}$} & \multirow[t]{4}{*}{0,4} & HA4 (Fatigabilité) & 11,45 & $<0,005$ & 0,33 \\
\hline & & NS4 (Manque d'ordre) & 7,98 & $<0,01$ & 0,22 \\
\hline & & C2 (Empathie) & 6,51 & $<0,02$ & $-0,21$ \\
\hline & & SD3 (Ressources personnelles) & 4,74 & $<0,04$ & $-0,22$ \\
\hline \multirow[t]{3}{*}{ Assujettissement (sb) } & \multirow[t]{3}{*}{0,27} & HA3 (Timidité avec les inconnus) & 7,25 & $<0,01$ & 0,26 \\
\hline & & SD1 (Responsabilité) & 7 & $<0,01$ & $-0,26$ \\
\hline & & SD4 (Acceptation de soi) & 6,93 & $<0,01$ & $-0,23$ \\
\hline \multirow[t]{2}{*}{ Abnégation (ss) } & \multirow[t]{2}{*}{0,16} & RD1 (Sentimentalité) & 8,97 & $<0,005$ & 0,29 \\
\hline & & C3 (Solidarité) & 5,5 & $<0,03$ & 0,22 \\
\hline \multirow{2}{*}{$\begin{array}{l}\text { Recherche d'approbation et de } \\
\text { reconnaissance (as) }\end{array}$} & 0,19 & SD4 (Acceptation de soi) & 13,89 & $<0,001$ & $-0,34$ \\
\hline & & SD1 (Responsabilité) & 5,84 & $<0,02$ & $-0,22$ \\
\hline Négativité/pessimisme (np) & 0,26 & SD4 (Acceptation de soi) & 7,17 & $<0,01$ & $-0,23$ \\
\hline & & HA1 (Inquiétude anticipatoire) & 5,81 & $<0,02$ & 0,27 \\
\hline & & HA4 (Fatigabilité) & 5,25 & $<0,03$ & 0,25 \\
\hline Sur-contrôle émotionnel (ei) & 0,24 & RD3 (Attachement) & 11,01 & $<0,005$ & $-0,32$ \\
\hline & & HA3 (Timidité avec les inconnus) & 8,38 & $<0,005$ & 0,28 \\
\hline Idéaux exigeants/critique & 0,18 & PS3 (Ambitieux) & 13,35 & $<0,001$ & 0,34 \\
\hline excessive (us) & & SD4 (Acceptation de soi) & 4,05 & $<0,05$ & $-0,19$ \\
\hline Punition (pu) & 0,11 & HA3 (Timidité avec les inconnus) & 13,86 & $<0,001$ & 0,35 \\
\hline
\end{tabular}

Tableau 4. Régression du YSQ-S3 sur les sous-dimensions (traits) du TCI-R. 


\section{Le pouvoir prédictif des dimensions du TCI-R}

Comme l'indique le tableau 3, chacun des 18 SPI du YSQ-S3 peut être prédit significativement $(p<0,05)$ par une ou plusieurs dimensions $d u$ TCI-R. Les modèles prédictifs permettent d'expliquer entre $6 \%$ et $34 \%$ ( $\mathrm{R}^{2}$ ajustés) de la variance des schémas considérés. On trouve les proportions de variance expliquée les plus élevées pour les 4 schémas suivants : contrôle de soi/autodiscipline insuffisants $\left(R^{2}=0,34\right)$, Isolement social $\left(R^{2}=0,32\right)$, échec $\left(R^{2}=0,29\right)$ et assujettissement $\left(R^{2}=0,28\right)$. En revanche, pour les schémas manque affectif et punition, les proportions de variance expliquée $\left(R^{2}\right)$ ne sont respectivement que de 0,09 et 0,06 .

Parmi les dimensions du TCI-R les plus prédictives des SPI, la dimension autodétermination apparaît un prédicteur significatif pour 12 SPI, l'évitement du danger l'est pour 6 schémas, la persistance pour 3 schémas et la dépendance à la récompense pour 2 schémas. Plusieurs dimensions du TCI-R ne contribuent à la prédiction d'aucun SPI. C'est le cas des dimensions recherche de nouveauté et transcendance. D'un autre côté, la dimension de coopération est un prédicteur significatif pour un seul schéma (abnégation).

\section{Le pouvoir prédictif des sous-dimensions du TCI-R}

Comme l'indique le tableau 4, les SPI du YSQ-S3 peuvent également être prédits de manière significative par des sous-dimensions du TCI-R. Les parts de variance expliquée ( $\mathrm{R}^{2}$ ajustés) sont ici comprises entre $10 \%$ et $40 \%$. On trouve les proportions de variance expliquée les plus élevées pour les 4 schémas suivants : contrôle de soi/autodiscipline insuffisants $\left(R^{2}=0,40\right)$, isolement social $\left(R^{2}=0,32\right)$, peur du danger ou de la maladie $\left(R^{2}=0,32\right)$ et échec $\left(R^{2}=0,30\right)$. En revanche, pour les schémas manque affectif et punition, les proportions de variance expliquée $\left(\mathrm{R}^{2}\right) \mathrm{s}$ 'établissent respectivement à seulement 0,10 et 0,11 .

Parmi les sous-dimensions du TCI-R les plus prédictives des SPI, la sous-dimension SD4 (acceptation de soi) apparaît comme un prédicteur significatif pour 7 schémas, HA4 (fatigabilité) pour 6 schémas, SD1 (responsabilité) pour 5 schémas et HA3 (timidité avec les inconnus) pour 4 schémas. D'un autre côté, plusieurs sous-dimensions du TCI-R ne contribuent à la prédiction d'aucun schéma. C'est le cas des sous-dimensions NS1 (excitabilité exploratoire), NS2 (impulsivité) et
NS3 (extravagance), HA2 (peur de l'incertain), RD4 (dépendance), PS4 (perfectionnisme), SD2 (buts dans la vie), C1 (tolérance sociale) et C4 (indulgence), ST2 (identification transpersonnelle) et ST3 (acceptation spirituelle).

\section{DISCUSSION}

\section{Les traits de personnalité comme marqueurs de troubles liés aux substances}

De manière générale, en comparaison avec les normes de référence, cette étude met en évidence des spécificités quant à la personnalité des patients ayant des troubles liés aux substances.

\section{YSQ-S3}

Pour ce qui est du YSQ-S3, cette étude met en évidence cinq SPI nettement suractivés chez les patients dépendants de substances : manque affectif, abandon/instabilité, abnégation, négativité/pessimisme et idéaux exigeants. D'autres études ont également mis en évidence une suractivation des SPI abandon/instabilité (Brotchie et coll., 2004 ; Roper et coll., 2010 ; Shorey et coll., 2013, 2014), manque affectif (Brotchie et coll., 2004 ; Grebot et Marchand, 2008 ; Roper et coll., 2010 ; Shorey et coll., 2013) et négativité/pessimisme (Shorey et coll., 2013, 2014). En revanche, contrairement à ce qui a été observé dans notre étude, les SPI abnégation et idéaux exigeants ne paraissent suractivés ni dans les deux études de Shorey et coll. $(2013,2014)$ ni dans celle de Roper et coll. (2010). D'un autre côté, les études de Brotchie et coll. (2004), de Roper et coll. (2010) et de Shorey et coll. (2013, 2014) mettent aussi à jour une suractivation des schémas de méfiance/abus, imperfection, dépendance, fusionnement, contrôle de soi insuffisant et peur du danger, lesquels schémas n'apparaissent pas suractivés dans notre étude (imperfection, dépendance et contrôle de soi insuffisant) ou alors seulement modérément, c'est-à-dire à un seuil de signification clinique situé entre $20 \%$ et $30 \%$ (méfiance/abus, fusionnement, peur du danger).

En somme, les études semblent converger sur la désignation des schémas de manque affectif et d'abandon/instabilité, comme problématiques sensibles dans les troubles liés aux substances. Les autres points sont sujets à divergences, des divergences qui pourraient provenir de différences dans la composition des échantillons (spécificité des comorbidités, nature des produits objets de la dépendance) ou de différences dans les instruments de mesure utilisés (différentes versions du YSQ 
ont été employées : YSQ- L2, YSQ-S2, YSQ-L3, YSQ-S3).

\section{TCI-R}

Pour le TCI-R, cette étude met en évidence une surpondération des notes d'évitement du danger et une sous-pondération des notes d'autodétermination chez les patients dépendants de substances. Ces observations sont en accord avec les résultats d'autres études portant sur des populations similaires (Abbate-Daga et coll., 2007 ; Basiaux et coll., 2001 ; Cohen et coll., 2005 ; Fassino et coll., 2004 ; Herrero et coll., 2008 ; Hosak et coll., 2004 ; Le Bon et coll., 2004). Dans la présente étude, on relève également une sous-pondération des notes de coopération, résultat corroboré par certaines études seulement (Abbate-Daga et coll., 2007 ; Herrero et coll., 2008) et une sous-pondération des notes de persistance, résultat qui semble, quant à lui, tout à fait propre à notre échantillon.

D'un autre côté, on ne constate pas ici d'élévation significative des scores globaux de recherche de nouveauté, contrairement à ce que suggèrent de nombreux auteurs, qui identifient la recherche de nouveauté comme une dimension fortement liée aux problèmes de substances (Abbate-Daga et coll., 2007 ; Basiaux et coll., 2001 ; Cohen et coll., 2005, Fassino et coll., 2004 ; Herrero et coll., 2008 ; Le Bon et coll., 2004 ; Lukasiewicz et coll., 2008 ; Prisciandaro et coll., 2012 ; Ravaja et coll., 2001). Seules, deux sous-dimensions de la recherche de nouveauté, NS2 (impulsivité) et NS3 (extravagance) apparaissent ici surpondérées par rapport aux normes. Relevons que, de l'avis de Cloninger (1987b), la recherche de nouveauté caractériserait surtout les problèmes addictifs de début précoce et à prépondérance masculine. Or, au vu du rapport hommes/femmes, près de 50/50 et d'un âge moyen de 45,5 ans, il est possible que ce type clinique ait été relativement sous-représenté dans notre échantillon.

Contrairement encore à ce qu'ont relevé d'autres études portant sur des personnes dépendantes aux substances (Abbate-Daga et coll., 2007 ; Fassino et coll., 2004 ; Herrero et coll., 2008 ; Hosak et coll., 2004 ; Le Bon et coll., 2004), nous n'observons pas, dans cet échantillon, d'élévation des notes de transcendance.

En somme, la propension à l'évitement du danger et le défaut d'autodétermination peuvent être considérés comme des problématiques sensibles chez les personnes présentant des difficultés liées aux substances. D'autres éléments, comme la recherche de nouveauté, le défaut de persistance, une faible aptitude à la coopération sociale et l'inclination à la transcendance de soi seraient, sans doute, davantage caractéristiques de certains sous-groupes, de certaines comorbidités et leur expression varierait selon la composition des échantillons.

\section{Limites}

Il importe de remarquer que des scores élevés en évitement du danger et des scores faibles en autodétermination sont des caractéristiques également partagées par des personnes présentant d'autres types de troubles psychiques, des troubles thymiques en particulier (Cloninger et coll., 2006; Loftus et coll., 2008 ; Nery et coll., 2008 ; Richter, Polak, Eisemann, 2003 ; Sayin, Kuruoglu, Yazici Gulec, Aslan, 2007), lesquels paraissent être une importante problématique comorbide dans notre échantillon. De même, des niveaux élevés d'activation de SPI, tels qu'évalués à l'aide de questionnaires YSQ, se retrouvent également dans d'autres types de troubles (Delattre et coll., 2004 ; Pinto-Gouveia, Castilho, Galhardo, Cunha, 2006 ; Unoka, Tolgyes, Czobor, 2007 ; Waller, Ohanian, Meyer, Osman, 2000), dans les troubles anxieux notamment et dans les problématiques de type état limite, difficultés que l'on sait assez présentes parmi les sujets de notre échantillon. Ceci pose la question de la spécificité de ces inventaires s'agissant d'identifier les facteurs de vulnérabilité à la dépendance aux substances. Ceci rappelle, par la même occasion, que les problèmes psychiques constituent également d'importants facteurs de risques addictifs.

Les modèles de Cloninger (1994) et de Young et coll. (2003) représentent différentes bases conceptuelles permettant d'examiner les antécédents développementaux de la dépendance aux substances. Les résultats de la présente étude ne se prêtent cependant pas à une interprétation causale. Il reste difficile de déterminer dans quelle mesure les traits mis en évidence reflètent des caractéristiques antérieures de la personnalité ou sont, plutôt, des conséquences des problèmes de dépendance. De fait, plusieurs études ont montré que l'abus de substances pouvait modifier en partie certains traits de personnalité. Par exemple, Berglund, Fahlke, Berggren, Eriksson, Balldin (2006) ont observé qu'une longue durée de consommation excessive d'alcool pouvait avoir une influence sur les traits de personnalité. Evren, Durkaya, Kose, Cetin, Dalbudak, Celik (2010) ont constaté que les notes de recherche de nouveauté diminuaient chez des alcooliques en traitement et 
augmentaient à nouveau en cas de rechute. Shorey, Anderson, Stuart (2014) ont indiqué, quant à eux, que les schémas précoces inadaptés de personnes dépendantes à l'alcool et aux opiacés, pouvaient être modifiés en suite d'un traitement. Roper et coll. (2010) et Hautekèete, Ameerudden, Eraldi-Gackière, Baert, Rusinek (2006) ont établi des constats similaires : une désactivation relative de certains SPI, en suite d'un traitement psychosocial de patients alcoolo-dépendants et une réactivation en cas de rechute. Une autre possibilité serait encore qu'il n'y ait pas de lien causal direct entre les problèmes liés à l'usage de substances et les traits de personnalité. Les uns et les autres pourraient être dus à une cause commune sous-jacente comme, par exemple, un déficit dans la régulation des émotions. Des études complémentaires seraient nécessaires, afin de mieux préciser la nature des relations entre personnalité et problèmes de substances.

\section{Explication des SPI par les données de tempéraments et de caractères}

Les analyses de régression montrent que les dimensions et les sous-dimensions du TCI-R permettent d'expliquer la variance de plusieurs SPI. Ce sont principalement les dimensions d'autodétermination et d'évitement du danger, qui ont une valeur prédictive pour pratiquement tous les SPI. Ces observations sont en accord avec celles d'Halvorsen et coll. (2009) qui, dans une étude mêlant sujets déprimés et non déprimés, mettaient en évidence des corrélations entre de nombreux SPI et les dimensions d'évitement du danger et d'autodétermination.

De manière plus fine, il apparaît que c'est surtout en sa valeur unitaire que l'autodétermination possède des propriétés prédictives alors que, du côté de l'évitement du danger, ce sont, plus spécifiquement, les sous-dimensions HA4 (fatigabilité), HA3 (timidité avec les inconnus) et HA1 (inquiétude anticipatoire) qui paraissent être des facteurs prédictifs.

Une faible autodétermination est corrélée à une large palette de SPI, dix au total. Par ordre décroissant d'importance, elle permet de prédire les schémas d'assujettissement, d'imperfection/ honte, d'abandon/instabilité, de droits personnels exagérés, de peur du danger ou de la maladie, de contrôle de soi insuffisant, de méfiance/abus, de fusionnement/personnalité atrophiée, de recherche d'approbation et d'échec. Il s'agit donc d'un prédicteur assez puissant, mais peu spécifique des SPI. Il apparaît corrélatif d'une certaine inadaptation globale des schémas cognitifs, mais pas vraiment d'un profil particulier. À l'opposé, l'inquiétude anticipatoire (HA1) apparaît fortement prédictive de deux schémas précis : la peur du danger ou de la maladie et l'abandon/instabilité, de façon assez spécifique. La fatigabilité (HA4) intervient dans les schémas de manque affectif, d'échec, de contrôle de soi insuffisant, de méfiance/abus et de négativité/pessimisme. Son pouvoir prédictif semble, cependant, beaucoup plus modeste, voire presque négligeable dans les deux derniers cas. Quant à la timidité (HA3), elle permet de prédire fort modestement les seuls schémas de punition, de sur-contrôle émotionnel et d'imperfection/honte. C'est assez limité. Enfin, toujours dans la gamme des contributions modestes, la dépendance à la récompense intervient dans l'explication des schémas de surcontrôle émotionnel, la persistance ou son défaut intervient dans l'explication des schémas d'idéaux exigeants, de dépendance/incompétence et de droits personnels exagérés, et la coopération dans celle des schémas d'abnégation de soi.

\section{CONCLUSIONS}

Les limites de notre échantillon, qui ne peut prétendre à la représentativité de la population des personnes dépendantes, invitent à ne retenir que les constats statistiquement les plus tangibles. Il semble possible de les résumer de la manière suivante : le développement de schémas cognitifs inadaptés peuvent certainement, comme l'ont suggéré Young et coll. (2003), être expliqués par des facteurs de personnalité sous-jacents et, en l'occurrence, par des « problèmes » de personnalité, tels qu'appréciés en tant qu'écarts aux normes des notes au TCI-R. Des notes faibles, dans la dimension d'autodétermination, surtout, permettent de prédire globalement la présence de schémas cognitifs dysfonctionnels. D'un autre côté, la particularité d'un profil TCI-R n'apparaît guère à même de caractériser un profil YSQ-S3 particulier. Les deux échelles sont, certes, toutes deux sensibles à la vulnérabilité psychique des personnes, mais elles expriment cette vulnérabilité de manière différente. En d'autres termes, elles sont structurellement non-redondantes et, donc, d'usage plutôt complémentaire. Quant aux éléments de vulnérabilité mis à jour dans cette étude, ils corroborent les constats généralement établis, à savoir que les tendances à l'évitement du danger et le défaut d'autodétermination, d'une part, et la sur-activation de SPI de manque affectif et d'abandon/instabilité, d'autre part, constituent des problématiques sensibles chez les personnes présentant des troubles liés à l'utilisation de subs- 
tances, sans pour autant qu'elles leur soient spécifiques. Il existe également certaines divergences entre les éléments de vulnérabilité mis à jour dans cette étude et ceux mis à jour dans d'autres études portant sur des populations réputées analogues, ces divergences touchent à la recherche de nouveauté et les SPI d'assujettissement et d'idéaux exigeants.

Ces éléments problématiques pourraient être le fait privilégié de sous-catégories spécifiques de troubles liés aux substances ou de certaines comorbidités susceptibles de varier d'un échantillon à l'autre.

\section{RÉFÉRENCES}

Abbate-Daga (Giovanni), Amianto (Federico), Rogna (Lorenzo), FAssino (Secondo).- Do anorectic men share personality traits with opiate dependent men? A case-control study, Addictive Behaviors, 32, 1, 2007, p. 170-174.

Ashton (Michael C.).- Individual differences and personality, New York, Elsevier Inc, 2013.

Ashton (Michael C.), Lee (Kibeom), Perugini (Marco), Szarota (Piotr), de Vries (Reinout E.), Di Blas (Lisa), Boies (Kathleen), De RAAD (Boele).- A six-factor structure of personality-descriptive adjectives: Solutions from psycholexical studies in seven languages, Journal of Personality and Social Psycholy, 86, 2, 2004, p. 356-66.

Ball (Samuel A.), Young (Jeffrey E.).- Dual focus schema therapy. A treatment manual for personality disorders and addictions, manuscrit non publié, 1998.

Basiaux (Philippe), Le Bon (Olivier), Dramaix (Michèle), Massat (Isabelle), Souery (Daniel), Mendlewicz (Julien), Pelc (Isidore), Verbanck (Paul).Temperament and Character Inventory (TCI) personality profile and sub-typing in alcoholic patients: A controlled study, Alcohol and Alcoholism, 36, 6, 2001, p. 584-587.

Berglund (Kristina), Fahlke (Claudia), Berggren (Ulf), ERIKsson (Matts), Balldin (Jan).- Personality profile in type I alcoholism: Long duration of alcohol intake and low serotonergic activity are predictive factors of anxiety proneness, Journal of Neural Transmission (Vienna), 113, 9, 2006, p. 1287-1298.

Bowden-Jones (Owen), IqBal (Muhammad Z.), Tyrer (Peter), SeIvewright (Nicholas), Cooper (Sylvia), Judd (Ali), Weaver (Tim) and Cosmic Study team.- Prevalence of personality disorder in alcohol and drug services and associated comorbidity, Addiction, 99, 10, 2004, p. 1306-1314.

Brotchie (Janet), Meyer (Caroline), Copello (Alex), Kidney (Robert), Waller (Glenn).- Cognitive representations in alcohol and opiate abuse: The role of core beliefs, British Journal of Clinical Psychology, 43, Pt 3, 2004, p. 337-342.

Cervone (Daniel), Pervin (Lawrence A.) - Personality: Theory and research, New York, Wiley and Sons, 2008.

Chakroun (Nadia), Doron (Jack), Swendsen (Joël).Consommation de substances psychoactives, problèmes affectifs et traits de personnalité : test de deux modèles d'association, Encéphale, 30, 6, 2004, p. 564-9.

Cloninger (C. Robert).- The genetic structure of personality and learning: A phylogenetic model, Clinical
Genetics, 46, 1 Spec No, 1994, p. 124-137.

Cloninger (C. Robert).- A unified biosocial theory of personality and its role in the development of anxiety states, Psychiatric Developments, 4, 3, 1986, p. 167-226.

Cloninger (C. Robert).- A systematic method for clinical description and classification of personality variants. A proposal, Archives of General Psychiatry, 44, 6, 1987a, p. 573-588.

Cloninger (C. Robert).- The Temperament and Character Inventory-Revised, St. Louis, MO, Center for Psychobiology of Personality, Washington University, 1999.

Cloninger (C. Robert).- Neurogenic adaptive mechanisms in alcoholism, Science, 236, 1987b, p. 410-416.

Cloninger (C. Robert), Sigvardsson (Soren), Gilligan (Shelia B.), von Knorring (Anne-Liis), Reich (Theodore), Bohman (Michael).- Genetic heterogeneity and the classification of alcoholism, Advances in Alcohol \& Substance Abuse, 7, 3-4, 1988, p. 3-16.

Cloninger (C. Robert), Svrakic (N. M.), SvrakiC (Dragan M.).- Role of personality self-organization in development of mental order and disorder, Development and Psychopathology, 9, 4, 1997, p. 881-906.

Cloninger (C. Robert), Svrakic (Dragan M.), PrzyBECK (Thomas R.).- A psychobiological model of temperament and character, Archives of General Psychiatry, 50, 1993, p. 975-990.

Cloninger (C. Robert), Svrakic (Dragan M.), Przybeck (Thomas R.).- Can personality assessment predict future depression? A twelve-month follow-up of 631 subjects, Journal of Affective Disorders, 92, 1, 2006, p. 35-44.

Cohen (Lisa J.), Gertmenian-KIng (Enid), Kunik (Lauren), Weaver (Carrie), London (Edythe D.), GALYNKER (Igor).- Personality measures in former heroin users receiving methadone or in protracted abstinence from opiates, Acta Psychiatrica Scandinavica, 112, 2, 2005, p. 149-158.

Compton (Wilson M.), Conway (Kevin P.), Stinson (Frederick S.), Colliver (James D.), Grant (Bridget F.).- Prevalence, correlates, and comorbidity of DSM-IV antisocial personality syndromes and alcohol and specific drug use disorders in the United States: Results from the national epidemiologic survey on alcohol and related conditions, Journal of Clinical Psychiatry, 66, 6, 2005, p. 677-685. 
Costa (Paul T.), McCrae (Robert R.).- From catalog to classification: Murray's need and the five-factor model, Journal of Personality and Social Psychology, 55, 2, 1988, p. 258-265.

Costa (Paul T.), McCrae (Robert R.).- Four ways five factors are basic, Personality and Individual Differences, 13, 6, 1992, p. 653-665.

CRAIG (Robert J.).-- Prevalence of Personality Disorders among Cocaine and Heroin Addicts, Substance Abuse, 21, 2, 2000, p. 87-94.

Decouvelaere (Florence), Graziani (Pierluigi), GackieRe-Eraldi (Daniela), Rusinek (Stéphane), Hautekèete (Marc).- Hypothèse de l'existence et de l'évolution de schémas cognitifs mal adaptés chez l'alcoolo-dépendant, Journal de thérapie comportementale et cognitive, 12, 2002, p. 43-48.

Delattre (Vanessa), Servant (Dominique), Rusinek (Stéphane), Lorette (C), Parquet (P. J.), Goudemand (Michel), Hautekèete (Marc).- Les schémas précoces dysfonctionnels : étude chez des patients adultes souffrant d'un trouble anxieux, Encéphale, 30, 3, 2004, p. 255-258.

Elkins (Irène J.), KInG (Serena M.), McGue (Matt), IACONO (William G.).- Personality traits and the development of nicotine, alcohol, and illicit drug disorders: Prospective links from adolescence to young adulthood, Journal of Abnormal Psychology, 115, 1, 2006, p. 26-39.

Evren (Cuneyt), Durkaya (Mine), Kose (Samet), Cetin (Rabia), Dalbudak (Ercan), Celik (Selime).- Novelty seeking among relapsed and non-relapsed male alcohol dependents during 12 month follow-up, Düşünen Adam, The journal of Psychiatry and Neurological Sciences, 23, 4, 2010, p. 249-255.

Fassino (Secondo), Abbate Daga (Giovanni), Delsedime (Nadia), Rogna (Lorenzo), Boggio (Sonia).Quality of life and personality disorders in heroin abusers, Drug Alcohol Depend, 76, 1, 2004, p. 73-80.

George (Susan M.), Connor (Jason P.), Gullo (Matthew J.), Young (Ross).-A prospective study of personality features predictive of early adolescent alcohol misuse, Personality and Individual Differences, 49, 3, 2010, p. 204-209.

Grant (Bridget F.), Stinson (Frederick S.), Dawson (Deborah A.), Chou (Patricia S.), Ruan (June W.), PickeRING (Roger P.). - Co-occurrence of 12-month alcohol and drug use disorders and personality disorders in the United States. Results from the national epidemiologic survey on alcohol and related conditions, Archives of General Psychiatry, 61, 2004, p. 361-368.

Grebot (Elisabeth), Marchand (Virgile).- Schémas précoces inadaptés chez des consommateurs de cannabis, Journal de thérapie comportementale et cognitive, 18, 2008, p. 98-103.

Halvorsen (Marianne), WANG (Catharina E.), Richter (Jörg), Myrland (Ine), Pedersen (Siv K.), Eisemann (Martin), Waterloo (Knut).- Early maladaptive schemas, temperament and character traits in clinically depressed and previously depressed subjects, Clinical Psychology \& Psychotherapy, 16, 5, 2009, p. 394-407.

Hansenne (Michel).- Psychologie de la personnalité, Paris, De Boeck, 2003.
Hansenne (Michel), Delhez (Marie), Cloninger (C. Robert).- Psychometric properties of the temperament and character inventory-revised (TCI-R) in a Belgian sample, Journal of Personality Assessment, 85, 1, 2005, p. $40-49$.

Hathaway (Starke R.), McKinley (John C.).- MMPI manual, New York, Psychological Corporation, 1943.

Hautekèete (Marc), Ameerudden (R), Eraldi-GacKIÈRE (Daniela), BAERT (Romain), Rusinek (Stéphane).L'évolution des schémas inadaptés durant et après une cure TCC chez des patients alcooliques qui rechutent ou ne rechutent pas : contribution à la théorie des schémas, Journal de therapie comportementale et cognitive, 16, 2006, p. 16-26.

Herrero (M. Jesus), Domingo-Salvany (Antonia), Torrens (Marta), Brugal (M. Teresa), Gutierrez (Fernando) and Itinere Investigators.- Personality profile in young current regular users of cocaine, Substance Use \& Misuse, 43, 10, 2008, p. 1378-1394.

Hosak (Ladislav), Preiss (Marek), Halir (Martin), Cermakova (Eva), Csemy (Ladislav).- Temperament and character inventory (TCI) personality profile in metamphetamine abusers: A controlled study, European Psychiatry, 19, 4, 2004, p. 193-195.

Howard (Matthew O.), Kivlahan (Daniel), Walker (Roger D.).- Cloninger's tridimensional theory of personality and psychopathology: Applications to substance use disorders, Journal of Studies on Alcohol, 58, 1, 1997, p. 48-66.

Katona (Zsuzsa), Kormendi (Attila).- Examination of the Young maladaptive schemes in a group of Gamblers Anonymous, Psychiatria Hungarica, 27, 6, 2012, p. 435-445.

Le Bon (Olivier), Basiaux (Philippe), Streel (Emmanuel), Tecco (Juan), HanaK (Catherine), Hansenne (Michel), Ansseau (Marc), Pelc (Isidore), Verbanck (Paul), Dupont (Sylviane).- Personality profile and drug of choice: A multivariate analysis using Cloninger's TCI on heroin addicts, alcoholics, and a random population group, Drug and Alcohol Dependence, 73, 2, 2004, p. 175-182.

LeE (Christopher W.), TAYLOR (Graham), DunN (John).Factor Structure of the Schema Questionnaire in a Large Clinical Sample, Cognitive Therapy and Research, 23, 4, 1999, p. 441-451.

Loftus (Shay T.), GARno (Jessica L.), JAEGER (Judith), Malhotra (Anil K.).- Temperament and character dimensions in bipolar I disorder: A comparison to healthy controls, Journal of Psychiatric Research, 42, 13, 2008, p. 1131-1136.

Lukasiewicz (Michael), Neveu (Xavier), Blecha (Lisa), Falissard (Bruno), Reynaud (Michel), Gasquet (Isabelle).- Pathways to substance-related disorder: A structural model approach exploring the influence of temperament, character, and childhood adversity in a national cohort of prisoners, Alcohol Alcohol, 43, 3, 2008, p. 287-295.

Masse (Louise C.), Tremblay (Richard E.).- Behavior of boys in kindergarten and the onset of substance abuse during adolescence, Archive of General Psychiatry, 54, 1, 1997, p. 62-68. 
McCRAe (Robert R.), Allik (Juri).- The Five-Factor Model of personality across cultures, New York, Kluwer, 2002.

Nery (Fabiano G.), Hatch (John P.), Glahn (David C.), Nicoletti (Mark A.), Monkul (E. Serap), Najt (Pablo), Fonseca (Manoela), Bowden (Charles L.), Cloninger (C. Robert), Soares (Jair C.).- Temperament and character traits in patients with bipolar disorder and associations with comorbid alcoholism or anxiety disorders, Journal of Psychiatric Research, 42, 7, 2008, p. 569-577.

Pallincourt (Romain), Kempeneers (Philippe), LeX (Olivier), Wagener (Luc), Parent (Marcel).- Hôpital de jour : outil de substitution dans l'alcoolo-dépendance, Revue des hôpitaux de jour psychiatriques et des thérapies institutionnelles, 11, 2009, p. 78-84.

Pelissolo (Antoine), LÉPINE (Jean-Pierre).- Normative data and factor structure of the Temperament and Character Inventory (TCI) in the French version, Psychiatry Research, 94, 1, 2000, p. 67-76.

Pinto-Gouveia (José), Castilho (Paula), Galhardo (Ana), Cunha (Marina).-- Early Maladaptive Schemas and Social Phobia, Cognitive Therapy and Research, 30, 5, 2006, p. 571-584.

Preuss (Ulrich W.), Johann (Monika), Fehr (Christoph), Koller (Gabi), Wodarz (Norbert), Hesselbrock (Victor), Wong (William M.), SoyKa (Michael).- Personality disorders in alcohol-dependent individuals: Relationship with alcohol dependence severity, European Addiction Research, 15, 4, 2009, p. 188-195.

Prisciandaro (James J.), Korte (Jeffrey E.), McRaeClark (Aimee L.), Brady (Kathleen T.).- Associations between behavioral disinhibition and cocaine use history in individuals with cocaine dependence, Addictive Behaviors, 37, 10, 2012, p. 1185-1188.

Ravaja (Niklas), Keltikangas-Jarvinen (Liisa).Cloninger's temperament and character dimensions in young adulthood and their relation to characteristics of parental alcohol use and smoking, Journal of Studies on Alcohol, 62, 1, 2001, p. 98-104.

Richter (Jörg), Polak (Thomas), Eisemann (Martin).Depressive mood and personality in terms of temperament and character among the normal population and depressive inpatients, Personality and Individual Differences, 35, 4, 2003, p. 917-927.

Roper (Louise), Dickson (Joanne M.), Tinwell (Claire), Bоoтн (Peter G.), McGuire (James).- Maladaptive Cognitive Schemas in Alcohol Dependence: Changes Associated with a Brief Residential Abstinence Program, Cognitive Therapy and Research, 34, 3, 2010, p. 207-215.

Sayin (Aslıhan), Kuruoglu (Aslı Ç.), Yazici Gulec (M.), AsLAN (Selçuk).- Relation of temperament and character properties with clinical presentation of bipolar disorder, Comprehensive Psychiatry, 48, 5, 2007, p. 446-451.

Schmidt (Norman B.), JoIner (Thomas E.), Young (Jeffery E.), Telch (Michael J.).- The schema questionnaire: Investigation of psychometric properties and the hierarchical structure of a measure of maladaptive schemas, Cognitive Therapy and Research, 19, 3, 1995, p. 295-321.

Shorey (Ryan C.), Anderson (Scott), Stuart (Gregory L.) - The relation between antisocial and borderline personality symptoms and early maladaptive schemas in a treatment seeking sample of male substance users, Clinical Psychology \& Psychotherapy, 21, 4, 2014, p. 341-351.

Shorey (Ryan C.), Stuart (Gregory L.), Anderson (Scott).- Early maladaptive schemas among young adult male substance abusers: A comparison with a non-clinical group, Journal of Substance Abuse Treatment, 44, 5, 2013, p. 522-527.

Shorey (Ryan C.), Stuart (Gregory L.), Anderson (Scott).- Differences in early maladaptive schemas between a sample of young adult female substance abusers and a non-clinical comparison group, Clinical Psychology \& Psychotherapy, 21, 1, 2014, p. 21-28.

Skeel (Reid L.), Pilarski (Carrie), Pytlak (Kimberley), NeudeCKER (John).- Personality and performance-based measures in the prediction of alcohol use, Psychology of Addictive Behaviors, 22, 3, 2008, p. 402-409.

Tellegen (Auke), Ben-Porath (Yossef S.), McNulty (John L.), Abrisi (Paul A.), Graham (John R.), Kaemmer (Beverly).- The MMPI-2 Restructured Clinical Scales: Development, validation and interpretation, Minneapolis, MN, University of Minnesota Press, 2003.

Unoka (Zsolt), Tolgyes (Tamas), Czobor (Pal).- Early maladaptive schemas and body mass index in subgroups of eating disorders: A differential association, Comprehensive Psychiatry, 48, 2, 2007, p. 199-204.

van Ammers (Edmond C.), Sellman (J. Douglas), Mulder (R. T.).- Temperament and Substance Abuse in Schizophrenia: Is There a Relationship?, The Journal of Nervous and Mental Disease, 185, 5, 1997, p. 283-288.

VERHEUL (Roel) - Co-morbidity of personality disorders in individuals with substance use disorders, European Psychiatry, 16, 5, 2001, p. 274-282.

Vukov (Mila), Baba-Milkic (Nada), Lecic (D.), Mujalkovic (Slobodan), Marinkovic (Jelena).- Personality dimensions of opiate addicts, Acta Psychiatrica Scandinavica, 91, 2, 1995, p. 103-107.

Waller (Glenn), Ohanian (Vartouhi), Meyer (Caroline), Osman (Selen).-- Cognitive content among bulimic women: The role of core beliefs, International Journal of Eating Disorders, 28, 2, 2000, p. 235-241.

Wills (Thomas A.), Vaccaro (Donato), McNamara (Grace).- Novelty seeking, risk taking, and related constructs as predictors of adolescent substance use: An application of Cloninger's theory, Journal of Substance Abuse, 6, 1, 1994, p. 1-20.

Young (Jeffrey E.).- Cognitive therapy for personality disorders, Sarasota, FL, Professional Resource Press, 1990.

Young (Jeffrey E.), Klosko (Janet S.), WeISHAAR (Marjorie E.).- Schema therapy: A practitioner's guide, New York, Guilford Press, 2003.

Young (Jeffrey E.), Klosko (Janet S.), WeISHAAR (Marjorie E.).- La thérapie des schémas, Bruxelles, De Boeck, 2005.

Young (Jeffrey E.), Pascal (Bernard), Cousineau (Pierre).-Questionnaire des schémas de Young (YSQ-S3), New York, Schema Therapy Institute, 2005. 